\title{
Obstructive sleep apnea, COPD, the overlap syndrome, and mortality: results from the 2005-2008 National Health and Nutrition Examination Survey
}

This article was published in the following Dove Press journal:

International Journal of COPD

\author{
Wei Du ${ }^{1, *}$ \\ Jun $\mathrm{Liu}^{2, *}$ \\ Jianlong Zhou ${ }^{1, *}$ \\ Dan Ye ${ }^{3}$ \\ Yan OuYang ${ }^{4}$ \\ Qingnan Deng' \\ 'Respiratory Diseases Group, the \\ 6th Unit, Department of Internal \\ Medicine, Guangzhou General \\ Hospital of Guangzhou Military \\ Command, Guangzhou 510010 , \\ China; ${ }^{2}$ Department of Gerontology, \\ Guangzhou General Hospital \\ of Guangzhou Military Command, \\ Guangzhou 510010, China; ${ }^{3}$ Luopu \\ Street Community Health Service \\ Centre, Guangzhou 5II43I, China; \\ ${ }^{4}$ Department of Respiratory Medicine, \\ Guangzhou General Hospital of \\ Guangzhou Military Command, \\ Guangzhou 510010, China \\ *These authors contributed equally \\ to this work
}

Objective: The aim of this study was to investigate the role of obstructive sleep apnea (OSA) on all-cause mortality in patients with COPD.

Methods: Data for this cross-sectional study were obtained from the National Health and Nutrition Examination Survey (NHANES) data (year 2005-2008). Eligible subjects were $\geq 20$ years who had no COPD or OSA $(n=9,237)$, had only OSA $(n=366)$, had only COPD $(n=695)$, and had OSA/COPD overlap syndrome $(\mathrm{n}=90)$. Univariate and multivariate analyses were used to evaluate factors associated with overall mortality.

Results: Multivariate analysis found that the COPD and OSA/COPD overlap syndrome groups had significantly higher chance of all-cause mortality than the group of subjects who did not have OSA or COPD (adjusted hazard ratio $[\mathrm{HR}]=1.5$ for the COPD group and 2.4 for the overlap syndrome group) $(P \leq 0.007)$. Although not significant, having OSA/COPD overlap syndrome was associated with higher likelihood of death than COPD alone (HR $=1.5 ; P=0.160)$. Other factors associated with higher overall mortality were aging, poorer family status, current smoker, serum vitamin D deficiency, cardiovascular disease, history of cancer, diabetes, and impaired renal function.

Conclusion: The present study found that COPD and OSA/COPD overlap syndrome were associated with higher all-cause mortality compared with patients without either disease and that OSA did not significantly increase mortality in patients with COPD.

Keywords: OSA, COPD, overlap syndrome, mortality

\section{Plain language summary}

The presence of obstructive sleep apnea (OSA) is a known common comorbidity of COPD. The relationship of the combination of OSA and COPD on mortality is not known. This study investigated the association of OSA, COPD, and OSA/COPD overlap syndrome with all-cause mortality. The study found that COPD and COPD with OSA overlap syndrome groups had significantly higher likelihood of death due to any cause than the group of subjects without either disease. Although not significant, having OSA/COPD overlap syndrome was associated with higher likelihood of all-cause mortality compared to COPD alone.

\section{Introduction}

Obstructive sleep apnea (OSA) and COPD are among the most common pulmonary diseases. ${ }^{1}$ OSA is estimated to affect $4 \%$ of men and $2 \%$ of women in the USA, suggesting that $\sim 10$ million people in the USA have the disease. ${ }^{2}$ OSA is characterized by recurring 
episodes of hypoxemia and apnea during sleep. ${ }^{3-5}$ COPD is typified by persistent airflow obstruction, irreversible pulmonary damage, and chronic pulmonary inflammation. COPD is also a systemic disease with nonpulmonary manifestations including depression, osteoporosis, anemia, and skeletalmuscle myopathy. ${ }^{6-8}$ COPD is a major cause of mortality and chronic disability and is predicted to become the third leading cause of death worldwide by $2020 .{ }^{9}$

COPD and OSA have common pathophysiology, for example, smoking. ${ }^{1}$ The two diseases are known to coexist, which was termed by Flenley ${ }^{10}$ as the "overlap syndrome". Flenley found that patients who had COPD with OSA had greater amount of nocturnal hypoxemia and hypercapnia than patients with COPD or OSA alone. Other studies have demonstrated that OSA/COPD overlap syndrome is associated with daytime oxygen desaturation, hypercapnia, and lower quality of life. ${ }^{11-13}$ Overlap syndrome is considered as a common disease, which may be different than the simple sum of OSA and COPD. ${ }^{1}$ The prevalence of COPD with OSA overlap syndrome is difficult to estimate as sleep disorders occur secondarily to COPD. ${ }^{14}$ A European-based study found that $\sim 1 \%$ of the total population and $9.2 \%$ of those with OSA had COPD as determined by spirometery. ${ }^{14}$

Mortality associated with OSA/COPD overlap syndrome has not been well studied. ${ }^{1,15,16}$ The aim of this cross-sectional study was to investigate the impact of OSA on the overall mortality in patients with COPD.

\section{Methods}

\section{Data source}

Data for this cross-sectional study were obtained from the National Health and Nutrition Examination Survey (NHANES) data (year 2005-2008) (Centers for Disease Control and Prevention [CDC], National Center for Health Statistics [NCHS], Hyattsville, MD, USA: US Department of Health and Human Services) (http://www.cdc.gov/nchs/ nhanes/). The survey is designed to evaluate the health and nutritional status of adults and children in the USA. It uses a complex, multistage design to collect and analyze data representative of the national, noninstitutionalized population of the USA (for further information about background, design, and operation of the survey, refer http://wwwn.cdc. gov/nchs/nhanes). The NCHS Research Ethics Review Board reviewed and approved data obtained from NHANES. All data obtained from NHANES data are de-identified, and consequently, analysis of the data does not require approval from the NCHS Research Ethics Review Board or written informed consent from subjects.
The mortality data of NHANES respondents were linked to National Death Index mortality data through December 31, 2011 (detail of this data linkage can be found at: $\underline{\text { https:// }}$ www.cdc.gov/nchs/data-linkage/mortality-public.htm).

\section{Study population}

We selected subjects between the age of $\geq 20$ years with known status of COPD and OSA within the selected year cycles. Participants with missing demographic data, COPD, sleep apnea status, or survival status were excluded. Subjects who had no COPD or OSA, had only OSA, had only COPD, and had OSA/COPD overlap syndrome were finally categorized. The presence of the disease was determined through answers to the following questions:

COPD: subjects who answered yes either to the question "Has a doctor or other health professional ever told you that you had chronic bronchitis?" or to the question "Has a doctor or other health professional ever told you that you had emphysema?" were defined as having COPD.

OSA: subjects who answered yes to the question "Have you ever been told by a doctor or other health professional that you have a sleep disorder?" and reported sleep apnea to "What was the sleep disorder?" were defined as having OSA.

OSA/COPD overlap syndrome: subjects who self-reported having both COPD and OSA were regarded as overlap.

\section{Study variables}

Independent variables included subjects with OSA, COPD, and OSA/COPD overlap syndrome. The remaining subjects were grouped into no COPD and no OSA and were considered the reference/control groups.

Subject-specific variables included demographics (age, gender, and race/ethnicity), socioeconomic status (family poverty income ratio and education level), lifestyles and behaviors (smoking history, alcohol consumption, physical activity [metabolic equivalent of task $\{$ MET $\}$ score]), selfreported medical condition (obesity, cardiovascular disease, diabetes, hypertension, dyslipidemia, renal insufficiency, and cancer), and serum vitamin D status.

Mortality was also assessed using linked mortality files, ${ }^{17}$ which allowed us to determine the major cause of death. The linked mortality files listed nine causes of death, with the remaining classified into "all other causes".

\section{Demographic data}

Age, gender, and race/ethnicity were recorded using interviewer-administered questionnaires (Demographic 
Variables and Sample Weights) from NHANES database. All participants were categorized into the following age groups: 20-39, 40-59, and 60-79 years. Race/ethnicity was self-reported as Mexican American, other Hispanic, non-Hispanic White, non-Hispanic Black, and other race, including multiracial. We further grouped the different race/ethnicity groups into the following four groups: nonHispanic White, Hispanic, non-Hispanic Black, and others (including Mexican American and other race).

\section{Socioeconomic status and education level}

Socioeconomic status was captured via the Family and Sample Person Demographics questionnaire, which was administered at the subject's home, by trained interviewers using the Computer-Assisted Personal Interviewing (CAPI) system. The ratio of family income to poverty refers to the ratio of family income to poverty threshold. The range of values includes $0-5$. We further classified them as $<1,1-2$, and $>2$.

Education level and the ratio of family income to poverty were recorded using interviewer-administered questionnaires (Demographic Variables and Sample Weights) from NHANES database.

\section{Lifestyles and behavioral variables}

Smoking status was recorded using interviewer-administered questionnaires (Smoking - Cigarettes Use) from NHANES database. Smoking was classified as never, former, and current smokers. Subjects who had $<100$ cigarettes in their life were defined as nonsmoker. Those who had at least 100 cigarettes but did not currently smoke were classified as former smoker. Those who response "yes" to the question "Do you smoke now?" were defined as current smoker. Alcohol consumption was classified as heavy drinker, moderate drinker, and lifetime abstainer. An average of $>14$ drinks per week for men, or $>7$ drinks per week for women, or $\geq 5$ drinks in a single day once or more, during the past year was defined as heavy drinker. An average of $\leq 14$ drinks per week for men, or $\leq 7$ drinks per week for women, or never $\geq 5$ drinks on a single day, during the past year was defined as moderate drinker. Subjects who reported $<12$ drinks ever were defined as lifetime abstainers. ${ }^{18}$

To estimate physical activity, we summed the product of weekly time spent in each activity reported by the participant multiplied by MET value for that activity yielding a metabolic equivalent-hour index. One MET is the energy expenditure of $1 \mathrm{kcal} / \mathrm{kg}$ body weight per hour. ${ }^{19}$ Those with $\geq 600 \mathrm{MET} \mathrm{min} /$ week were referred to as physically active, while those with $<600 \mathrm{MET} \mathrm{min} /$ week were referred to as less than active based on the World Health Organization recommendation. ${ }^{20-22}$

\section{Diseases association}

Different medical conditions were self-reported using interviewer-administered questionnaires (Medical Conditions) from NHANES database. ${ }^{22}$ In this study, disease variables included obesity, cardiovascular disease, diabetes, hypertension, renal insufficiency, and dyslipidemia.

From examination and laboratory data, we assessed body mass index and defined obesity using the standard body mass index (BMI) category. Those who had $\mathrm{BMI} \geq 30 \mathrm{~kg} / \mathrm{m}^{2}$ were referred to as obese, those who had BMI $\geq 25$ to $<30 \mathrm{~kg} / \mathrm{m}^{2}$ were referred to as overweight, and those who had BMI $<25 \mathrm{~kg} / \mathrm{m}^{2}$ were referred to as underweight/normal weight group.

Cardiovascular disease (including coronary heart disease, angina, congestive heart failure, myocardial infarction, and stroke) and cancer history were defined by the question "Has a doctor or other health professional ever told you that you have (disease)". Diabetes mellitus was defined as a self-report or having been told by a doctor or health professional that a subject had diabetes or sugar diabetes, or the person was currently taking diabetic pills or insulin. Hypertension was defined by those who responded yes to the questions "Were you told on $\geq 2$ different visits that you had hypertension, also called high blood pressure?" or "Because of your (high blood pressure/hypertension), have you ever been told to take prescribed medicine?" Dyslipidemia was defined by self-report of having been told to take prescription for lowering blood lipid, or the subjects had one of the following: serum total cholesterol $>200 \mathrm{mg} / \mathrm{dL}$, serum triglyceride $>200 \mathrm{mg} / \mathrm{dL}$, and serum $\mathrm{HDL}<40 \mathrm{mg} / \mathrm{dL}$ (information from laboratory measurement data files).

Renal insufficiency was interpreted with the use of estimated glomerular filtration rate (eGFR) using the following equation: ${ }^{23}$

$$
\begin{aligned}
\mathrm{eGFR}= & 175 \times(\text { Standardized })^{-1.154} \\
& \times(\text { serum creatinine age })^{-0.203} \times(0.742 \text { if female }) \\
& \times(1.210 \text { if non-Hispanic Black })
\end{aligned}
$$

We categorized the findings into eGFR $<60$ and $\geq 60 \mathrm{~mL} /$ $\min / 1.73 \mathrm{~m}^{2}$. We used $60 \mathrm{~mL} / \mathrm{min} / 1.73 \mathrm{~m}^{2}$ as the cutoff for eGFR; $\geq 60 \mathrm{~mL} / \mathrm{min} / 1.73 \mathrm{~m}^{2}$ was classified as stages 1 and 2 and, in general, was not associated with signs and symptoms of insufficient kidney function. 


\section{Serum vitamin $D$ level}

Serum 25-hydroxyvitamin D data were extracted based on "Vitamin D" from NHANES Laboratory Data Protocol (detailed description of laboratory procedure manual can be found at NHANES web page: https://wwwn.cdc.gov/ Nchs/Nhanes/2005-2006/VID D.htm\#LBDVIDMS). Normal vitamin D serum levels were defined as $>75 \mathrm{nmol} / \mathrm{L}$ (30 ng/mL), vitamin D insufficiency was defined as 50-75 nmol/L (20-30 ng/mL), and vitamin D deficiency was defined as $<50 \mathrm{nmol} / \mathrm{L}(20 \mathrm{ng} / \mathrm{mL}){ }^{24}$

\section{Statistical analysis}

The primary endpoint was all-cause mortality.

All categorical variables were shown as unweighted counts (weighted \%). Chi-square test was conducted to determine differences in categorical variables. Univariate complex samples Cox regression analysis was performed to determine the risk factors for overall mortality. Multivariate Cox regression analysis was simultaneously adjusted for different disease entities, age, race, poverty income ratio, education, smoking, alcohol consuming, physical activity, serum vitamin D level, cancer history, cardiovascular disease, diabetes mellitus, hypertension, and eGFR.

All analyses included special sample weights, stratum, and primary sampling units (PSU) as per recommendations from the NCHS and complex sample analysis to address oversampling, nonresponse, and noncoverage and to provide nationally representative estimates. All statistical assessments were two sided and evaluated at the 0.05 level of significance. Statistical analyses were performed using the IBM SPSS statistical software Version 22 for Windows (IBM Corporation, Armonk, NY, USA).

\section{Results}

\section{Subject characteristics}

A total of 20,739 participants were included in the NHANES 2005-2008 cycle. Of these participants, 10,914 NHANES adult participants ( $\geq 20$ years) who were examined at a mobile examination center (MEC) were further evaluated for inclusion into the study. After excluding 48 subjects with the unknown status of OSA, 39 subjects with the unknown COPD status, 11 subjects who did not have mortality follow-up, and 428 subjects who missed NHANES sample weight, 10,388 participants were included in the present analysis.

Using NHANES sample weight, the analytic sample size of 10,388 subjects was equivalent to a population-based sample size of $210,916,313$ participants (Table 1). Of the 10,388 individuals, 9,237 (88.9\%) subjects had neither COPD nor OSA, 366 (3.8\%) subjects had OSA alone, 695 (6.5\%)

Table I Subject characteristics (unweighted $n=10,388$, weighted $n=2|0,9| 6,3 \mid 3$ )

\begin{tabular}{|c|c|c|c|c|c|c|}
\hline Characteristics & $\begin{array}{l}\text { Total } \\
(n=\mid 0,388)\end{array}$ & $\begin{array}{l}\text { Neither COPD } \\
\text { nor OSA }(n=9,237)\end{array}$ & $\begin{array}{l}\text { OSA alone } \\
(n=366)\end{array}$ & $\begin{array}{l}\text { COPD alone } \\
(n=695)\end{array}$ & $\begin{array}{l}\text { OSA/COPD } \\
\text { overlap }(n=90)\end{array}$ & P-value \\
\hline \multicolumn{7}{|c|}{ Demographic and socioeconomic status } \\
\hline Age (year) & $46.6 \pm 0.4$ & $45.8 \pm 0.4$ & $52.7 \pm 0.8$ & $53.9 \pm 0.7$ & $55.8 \pm 1.5$ & $<0.00 I^{*}$ \\
\hline $20-39$ & $3,664(37.8)$ & $3,474(40.3)$ & I 19 (20.3) & $63(16.1)$ & $8(9.9)$ & \\
\hline $40-59$ & $3,235(38.9)$ & $2,827(38.1)$ & $225(41.0)$ & 149 (53.2) & $34(46.4)$ & \\
\hline $60-79$ & $2,755(18.9)$ & $2,305(17.4)$ & $27 \mid(31.6)$ & $136(27.6)$ & $43(40.6)$ & \\
\hline $80+$ & $734(4.4)$ & $631(4.3)$ & $80(7.0)$ & $18(3.0)$ & $5(3.1)$ & \\
\hline Gender & & & & & & $<0.001^{*}$ \\
\hline Female & $5,354(5 \mid .9)$ & $4,788(51.9)$ & $|2|(3 \mid .4)$ & $405(63.1)$ & $40(50.6)$ & \\
\hline Male & $5,034(48.1)$ & $4,449(48.1)$ & $245(68.6)$ & $290(36.9)$ & $50(49.4)$ & \\
\hline Race & & & & & & $<0.00$ I* \\
\hline Non-Hispanic White & $5,007(70.6)$ & $4,278(69.5)$ & $224(80.9)$ & $455(80.6)$ & $50(7 \mid .2)$ & \\
\hline Non-Hispanic Black & $2,247(I I .3)$ & $2,029(11.6)$ & $80(9.4)$ & $112(7.9)$ & $26(15.5)$ & \\
\hline Mexican American & $1,936(8.2)$ & I,84I (8.8) & $35(3.9)$ & $54(2.9)$ & $6(2.5)$ & \\
\hline Other Hispanic & $777(4.1)$ & $707(4.3)$ & $18(3.3)$ & $48(3.0)$ & $4(1.5)$ & \\
\hline Other race & $421(5.7)$ & $382(5.8)$ & $9(2.6)$ & $26(5.5)$ & $4(9.3)$ & \\
\hline Poverty income ratio & & & & & & $<0.001^{*}$ \\
\hline$>2$ & $5,228(63.0)$ & $4,688(63.5)$ & $217(70.6)$ & $280(52.2)$ & $43(55.9)$ & \\
\hline $\mathrm{I}-2$ & $2,579(19.5)$ & $2,261(19.2)$ & $78(15.4)$ & $220(25.9)$ & $20(20.1)$ & \\
\hline$<1$ & I,843 (I I.8) & I,624 (II.6) & $47(8.6)$ & $150(15.6)$ & $22(18.1)$ & \\
\hline Education & & & & & & $<0.00 I^{*}$ \\
\hline Ever attend college & $4,780(55.6)$ & $4,246(55.8)$ & $200(6 \mathrm{I} .4)$ & $287(48.5)$ & $47(6 \mathrm{I} .7)$ & \\
\hline Never attend college & $5,595(44.3)$ & $4,980(44.1)$ & $166(38.6)$ & 406 (5I.3) & $43(38.3)$ & \\
\hline
\end{tabular}


Table I (Continued)

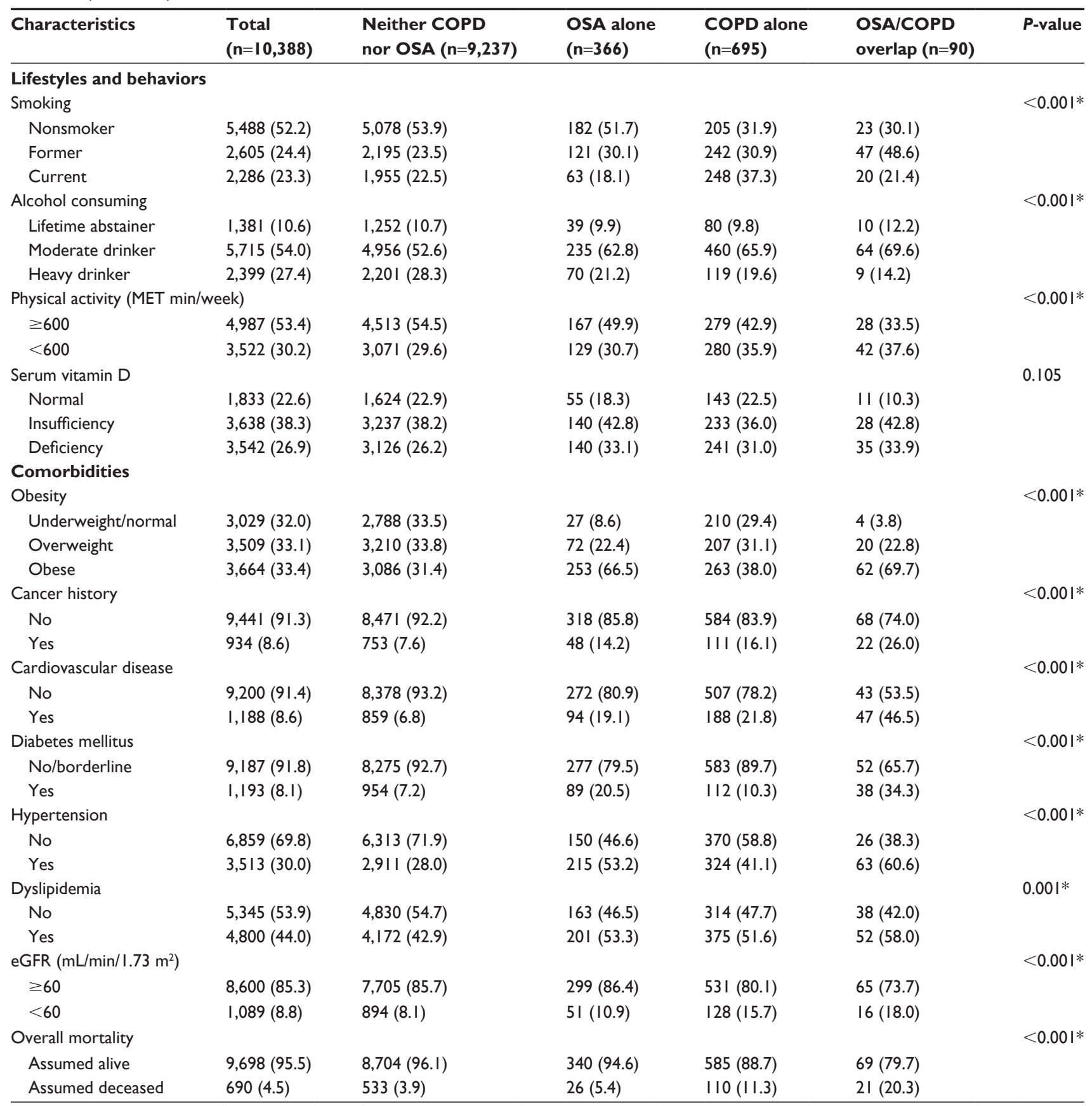

Notes: Categorical variables were shown unweighted counts (weighted \%). Chi-square test was conducted to determine differences in categorical variables. $*$ Significant difference between groups, $P$-value $<0.05$.

Abbreviations: eGFR, estimated glomerular filtration rate; MET, metabolic equivalent of task; OSA, obstructive sleep apnea.

subjects had COPD alone, and $90(0.8 \%)$ subjects had OSA/ COPD overlap syndrome.

The subject groups differed across demographic characteristics (Table 1). The patients with OSA/COPD overlap syndrome had higher mean age than those with COPD alone, OSA alone, or neither disease. A higher proportion of males had OSA alone (68.6\%) and most women had COPD alone $(63.1 \%)(P<0.001)$. Non-Hispanic Blacks had a significantly higher proportion of OSA/COPD overlap syndrome than neither disease, OSA alone, or COPD alone $(P<0.001)$, as also did poorer families (poverty income ratio $<1)(P<0.001)$. OSA/COPD overlap syndrome was observed in a higher percentage of subjects who formerly smoked, were moderate drinkers, and had $<600 \mathrm{MET} \mathrm{min} /$ week physical activity $(P<0.001)$. In addition, a higher percentage of subjects with OSA/COPD overlap syndrome were obese and had cancer 
history, cardiovascular disease, diabetes mellitus, hypertension, or dyslipidemia compared with the other groups $(P \leq 0.001)$.

The majority of the subjects in the present study were assumed to be alive at the end of the survey period ( $\geq 79.7 \%$ ); however, a higher mortality of subjects with OSA/COPD overlap syndrome was observed compared with the other groups $(P<0.001)$.
Overall mortality

The results from univariate and multivariate Cox regression analyses of overall mortality are shown in Table 2. Multivariate analysis found that the overall risk of death was significantly higher in patients with COPD alone and OSA/COPD overlap syndrome compared with patients who did not have either disease (adjusted hazard ratio $[\mathrm{HR}]=1.5$ for

Table 2 Cox regression analyses to determine the factors associated with overall mortality

\begin{tabular}{|c|c|c|c|c|}
\hline \multirow[t]{2}{*}{ Variable } & \multicolumn{2}{|l|}{ Univariate } & \multicolumn{2}{|l|}{ Multivariate } \\
\hline & HR (95\% Cl) & $P$-value & $\operatorname{aHR}(95 \% \mathrm{Cl})$ & $P$-value \\
\hline \multicolumn{5}{|l|}{ Disease entity } \\
\hline OSA alone vs neither COPD nor OSA & $1.5(0.9-2.3)$ & 0.113 & I.I (0.7-I.8) & 0.566 \\
\hline COPD alone vs neither COPD nor OSA & $3.1(2.4-4.0)$ & $<0.00 I^{*}$ & $1.5(1.2-2.0)$ & $0.001 *$ \\
\hline OSA/COPD overlap vs neither COPD nor OSA & $5.8(3.2-10.8)$ & $<0.00 I^{*}$ & $2.4(1.3-4.3)$ & $0.007^{*}$ \\
\hline OSA/COPD overlap vs COPD alone & $1.9(1.1-3.2)$ & $0.018^{*}$ & $1.5(0.8-2.8)$ & 0.160 \\
\hline \multicolumn{5}{|l|}{ Demographic and socioeconomic status } \\
\hline \multicolumn{5}{|l|}{ Age (years) } \\
\hline $20-39$ & Reference & & Reference & \\
\hline $40-59$ & $3.6(2.1-6.3)$ & $<0.00 I^{*}$ & $2.9(1.7-5.1)$ & $<0.00 I^{*}$ \\
\hline $60-79$ & $16.6(10.3-26.9)$ & $<0.00 I^{*}$ & $9.1(5.5-14.9)$ & $<0.001 *$ \\
\hline $80+$ & $74.4(47.2-1 \mid 7.3)$ & $<0.00 I^{*}$ & $32.0(18.8-54.3)$ & $<0.00 I^{*}$ \\
\hline \multicolumn{5}{|l|}{ Gender } \\
\hline Female & Reference & & & \\
\hline Male & I.I (0.9-I.3) & 0.407 & & \\
\hline \multicolumn{5}{|l|}{ Race } \\
\hline Non-Hispanic White & Reference & & Reference & \\
\hline Non-Hispanic Black & $1.0(0.8-1.2)$ & 0.935 & $1.0(0.8-1.3)$ & 0.708 \\
\hline Mexican American & $0.5(0.3-0.6)$ & $<0.00 I^{*}$ & $0.8(0.6-1.1)$ & 0.246 \\
\hline Other Hispanic & $0.4(0.2-0.8)$ & $0.016^{*}$ & $0.6(0.3-1.3)$ & 0.214 \\
\hline Other race & $0.4(0.2-0.9)$ & $0.038^{*}$ & $0.7(0.3-1.5)$ & 0.338 \\
\hline \multicolumn{5}{|l|}{ Poverty income ratio } \\
\hline$>2$ & Reference & & Reference & \\
\hline $\mathrm{I}-2$ & $2.4(1.9-3.1)$ & $<0.00 I^{*}$ & $1.3(1.0-1.7)$ & $0.040 *$ \\
\hline$<1$ & I.8 (I.3-2.5) & $0.002 *$ & $1.3(0.9-1.9)$ & 0.120 \\
\hline \multicolumn{5}{|l|}{ Education } \\
\hline Ever attend college & Reference & & Reference & \\
\hline Never attend college & $2.2(1.7-2.8)$ & $<0.00 I^{*}$ & $1.2(0.9-1.5)$ & 0.172 \\
\hline \multicolumn{5}{|l|}{ Lifestyles and behaviors } \\
\hline \multicolumn{5}{|l|}{ Smoking } \\
\hline Nonsmoker & Reference & & Reference & \\
\hline Former & $2.0(1.6-2.5)$ & $<0.00 I^{*}$ & $1.3(1.0-1.8)$ & 0.052 \\
\hline Current & $1.2(0.9-1.7)$ & 0.177 & $2.0(1.4-3.0)$ & $0.001 *$ \\
\hline \multicolumn{5}{|l|}{ Alcohol consuming } \\
\hline Lifetime abstainer & Reference & & Reference & \\
\hline Moderate drinker & $0.7(0.5-1.0)$ & 0.103 & $0.9(0.7-1.3)$ & 0.673 \\
\hline Heavy drinker & $0.3(0.2-0.4)$ & $<0.00 I^{*}$ & $0.8(0.6-1.2)$ & 0.291 \\
\hline \multicolumn{5}{|l|}{ Physical activity (MET min/week) } \\
\hline$\geq 600$ & Reference & & Reference & \\
\hline$<600$ & $2.5(1.9-3.4)$ & $<0.00 I^{*}$ & $1.3(1.0-1.8)$ & 0.097 \\
\hline \multicolumn{5}{|l|}{ Serum vitamin $D$} \\
\hline Normal & Reference & & Reference & \\
\hline Insufficiency & $1.2(0.9-1.5)$ & 0.238 & I.I (0.8-I.4) & 0.595 \\
\hline Deficiency & $1.8(1.3-2.6)$ & $0.001 *$ & I.5 (I.I-2.I) & $0.01 I^{*}$ \\
\hline
\end{tabular}


Table 2 (Continued)

\begin{tabular}{|c|c|c|c|c|}
\hline \multirow[t]{2}{*}{ Variable } & \multicolumn{2}{|l|}{ Univariate } & \multicolumn{2}{|l|}{ Multivariate } \\
\hline & HR (95\% Cl) & $P$-value & aHR $(95 \% \mathrm{Cl})$ & $P$-value \\
\hline \multicolumn{5}{|l|}{ Comorbidities } \\
\hline \multicolumn{5}{|l|}{ Obesity } \\
\hline Underweight/normal & Reference & & & \\
\hline Overweight & $0.9(0.7-1.1)$ & 0.276 & & \\
\hline Obese & $0.8(0.5-1.1)$ & 0.120 & & \\
\hline \multicolumn{5}{|l|}{ Cancer history } \\
\hline No & Reference & & Reference & \\
\hline Yes & $4.2(3.4-5.1)$ & $<0.00 I^{*}$ & $1.6(1.3-1.9)$ & $<0.00 I^{*}$ \\
\hline \multicolumn{5}{|l|}{ Cardiovascular disease } \\
\hline No & Reference & & Reference & \\
\hline Yes & $7.9(6.3-9.9)$ & $<0.00 I^{*}$ & $1.9(1.5-2.4)$ & $<0.00 I^{*}$ \\
\hline \multicolumn{5}{|l|}{ Diabetes mellitus } \\
\hline No/borderline & Reference & & Reference & \\
\hline Yes & $3.9(3.1-4.8)$ & $<0.00 I^{*}$ & $1.6(1.2-2.1)$ & $0.002 *$ \\
\hline \multicolumn{5}{|l|}{ Hypertension } \\
\hline No & Reference & & Reference & \\
\hline Yes & $3.1(2.4-4.0)$ & $<0.00 I^{*}$ & $1.0(0.8-1.3)$ & 0.744 \\
\hline \multicolumn{5}{|l|}{ Dyslipidemia } \\
\hline No & Reference & & & \\
\hline Yes & I.I (0.9-I.3) & 0.451 & & \\
\hline \multicolumn{5}{|l|}{ eGFR $\left(\mathrm{mL} / \mathrm{min} / \mathrm{l} .73 \mathrm{~m}^{2}\right)$} \\
\hline$\geq 60$ & Reference & & Reference & \\
\hline$<60$ & $6.6(5.5-8.0)$ & $<0.00 I^{*}$ & $1.4(1.1-1.9)$ & $0.006 *$ \\
\hline
\end{tabular}

Note: *Statistically significant, $P$-value $<0.05$.

Abbreviations: aHR, adjusted hazard ratio; $\mathrm{Cl}$, confidence interval; eGFR, estimated glomerular filtration rate; HR, hazard ratio; MET, metabolic equivalent of task; OSA, obstructive sleep apnea.

COPD alone and $\mathrm{HR}=2.4$ for OSA/COPD overlap syndrome; $P$-values $\leq 0.007$ ) (Figure 1). In addition, the likelihood of death was higher in the OSA/COPD overlap syndrome group compared with the COPD alone group; although this did not reach statistical significance (adjusted $\mathrm{HR}=1.5, P=0.160$ ).

Other factors associated with higher overall mortality were aging (40-59 years, adjusted HR $=2.9 ; 60-79$ years,

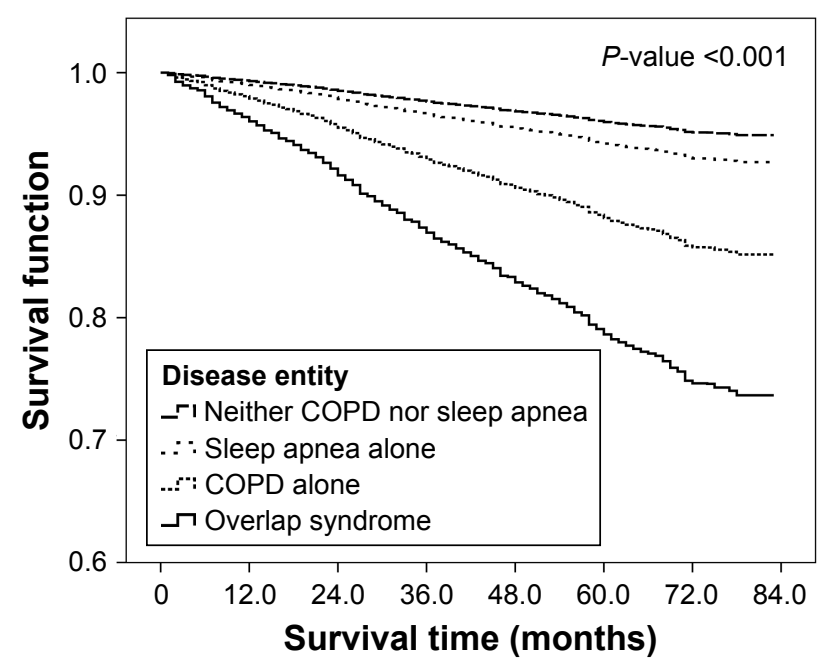

Figure I The cumulative survival distribution of disease entity. adjusted $\mathrm{HR}=9.1 ; 80$ years and older, adjusted $\mathrm{HR}=32.0$; all $P<0.001$ ), poorer family status ( $\leq 2$ poverty income ratio) (adjusted $\mathrm{HR}=1.3$ for the poverty income ratio of $1-2$; $P \leq 0.040$ ), current smoker (adjusted HR $=2.0 ; P=0.001$ ), serum vitamin $\mathrm{D}$ deficiency (adjusted $\mathrm{HR}=1.5, P=0.011$ ), cancer history (adjusted HR $=1.6 ; P<0.001$ ), cardiovascular disease (adjusted HR $=1.9 ; P<0.001$ ), diabetes mellitus (adjusted HR $=1.6 ; P=0.002$ ), and impaired renal function, defined as eGFR $<60 \mathrm{~mL} / \mathrm{min} / 1.73 \mathrm{~m}^{2}$ (adjusted $\mathrm{HR}=1.4$, $P=0.006)$.

\section{Discussion}

The presence of sleep apnea is acknowledged as a common comorbidity of COPD. ${ }^{1}$ The relationship of the combination of OSA and COPD on overall mortality is not well understood. The current study investigated the role of OSA on all-cause mortality in patients with COPD. Multivariate analysis found that subjects with COPD or OSA/COPD overlap syndrome had significantly higher likelihood of death than the group of subjects without either disease. Although not significant, having OSA/COPD overlap syndrome was associated with higher mortality than COPD. Aging, poorer family status, current smoker, serum vitamin D deficiency, 
cardiovascular disease, history of cancer, diabetes, and impaired renal function were found to be associated with overall mortality, independent of COPD or OSA status.

Prior studies have evaluated factors associated with mortality in patients with COPD, OSA, or OSA/COPD overlap syndrome. ${ }^{25-31}$ OSA has been associated with increased chance of a number of serious adverse cardiovascular events that can result in death, such as pulmonary and systemic hypertension, arrhythmia, myocardial ischemia/infarction, stroke, and heart failure. ${ }^{28-30}$ In addition, chronic kidney disease has been found to be significantly associated with OSA in a large population study of men. ${ }^{32}$ Important risk factors for death in patients with COPD include cancer and cardiovascular disease. ${ }^{25}$ In addition, COPD exacerbations increase mortality. ${ }^{26,33}$ Other comorbidities associated with COPD that can impact health status and prognosis include metabolic disorder, osteoporosis, anxiety, depression, skeletal muscle dysfunction, cachexia, gastrointestinal disease, and other respiratory conditions. ${ }^{34}$

Mortality data for patients who had OSA/COPD overlap syndrome are limited. Several studies have investigated the clinical effects of OSA/COPD on clinical outcomes. OSA/ COPD overlap syndrome is with excess mortality, commonly from cardiovascular causes, which is greater than that attributable to the individual disease. ${ }^{1,15,35}$ Marin et al ${ }^{15}$ showed that patients with OSA/COPD overlap syndrome had increased mortality and also significantly worse quality of life when compared with COPD controls. Interestingly, many COPD patients, particularly the "blue-bloaters", die disproportionately at night. ${ }^{25}$ It is possible the blue-bloaters may have overlap syndrome. ${ }^{1}$ Prior studies indicate that OSA increases the likelihood of mortality in patients with COPD. Marin et $\mathrm{al}^{15}$ in a longitudinal study observed that over 9 years, all cause-mortality was greater in patients with OSA/COPD overlap syndrome not treated with continuous positive airway pressure (CPAP) (42.2\%) compared with those that received CPAP therapy $(24.2 \%)$. The cause of the increased mortality with the lack of CPAP treatment may result from cardiovascular disease due to pulmonary hypertension and cardiac arrhythmia resulting from prolonged hypoxemia. ${ }^{1,11,15}$ A systematic review by Shawon et al (2016), ${ }^{36}$ which included 27 studies, found that OSA/ COPD overlap syndrome was prevalent in $\sim 2.9 \%-65.9 \%$ of patients with COPD. They found that overlap syndrome patients had greater nocturnal oxygen desaturation (oxygen saturation level $\leq 90 \%$ ) and worse sleep quality compared with patients with only OSA. In addition, overlap syndrome was associated with more frequent cardiovascular morbidity, poorer quality of life, more frequency COPD exacerbations, and higher hospital costs.
The underlying mechanism by which OSA/COPD overlap syndrome increases morbidity and mortality is not clear. ${ }^{1}$ Prolonged hypoxia and nighttime hypercapnia may increase the risk of death. ${ }^{1}$ In addition, both diseases have systemic impacts; both cause inflammation and oxidative stress. ${ }^{1}$ It is possible that both inflammatory and oxidative stress pathways together are critical to the increased morbidity and mortality seen in patients with OSA/COPD overlap syndrome. ${ }^{37}$

The current study has several strengths. NHANES is a benchmark national health survey and is one of the few population-based surveys that include validated examination measures, biological specimen collection, and limited measures of health status. Rigorous training in recruitment and data collection ensures high response rates, national representativeness, and high-quality data collection. The sample size was sufficiently large to allow accurate evaluation of prevalence measures at the national level. The fact that the database includes a large multiethnic population sample allows the exploration of racial/ethnic differences in identifying factors associated with mortality in COPD patients and COPD patients with OSA overlap syndrome.

The study also has some limitations. The study was cross-sectional in design, and consequently, it is not possible to infer any causal relationships. The sample of subjects included is not representative of the USA. The sample selected was to be demographically representative, but because the two teams only visit a total of 16 places a year, it is impossible to achieve good geographic coverage. Therefore, the survey is best used for national data or maybe for very large states or groups of states. The survey data are also not good for looking at changes over time because one does not know if observed changes result from geographic irregularities of the survey. Interview (questionnaire) data are based on self-reports, as the classifications of OSA, COPD, and overlap syndrome, and are therefore subjective to recall problems, misunderstanding of the question, and a variety of other factors. To overcome these biases, we chose objective laboratory data or body measures to replace those variables highly subjective to personal recall. NHANES is US data (including representative proportions of people of different ethnicity), and the findings should be validated in other countries.

\section{Conclusion}

The present study found that patients with COPD and OSA/ COPD overlap syndrome had significantly higher likelihood of death due to any cause compared to patients without either disease. In addition, the likelihood of all-cause mortality was 
higher in the OSA/COPD overlap syndrome compared to COPD alone group; however, this did not reach statistical significance.

\section{Acknowledgments}

We thank Yan Wang for technical assistance and Wenjie Huang and Zhenhui Guo for discussion. This study was supported by the Medical Science and Technology Foundation of Guangdong, China (no A2015240).

\section{Disclosure}

The authors report no conflicts of interest in this work.

\section{References}

1. Owens RL, Malhotra A. Sleep-disordered breathing and COPD: the overlap syndrome. Respir Care. 2010;55(10):1333-1344. Discussion 44-46.

2. Young T, Palta M, Dempsey J, Skatrud J, Weber S, Badr S. The occurrence of sleep-disordered breathing among middle-aged adults. $N$ Engl J Med. 1993;328(17):1230-1235.

3. Punjabi NM. The epidemiology of adult obstructive sleep apnea. Proc Am Thorac Soc. 2008;5(2):136-143.

4. Peppard PE, Young T, Barnet JH, Palta M, Hagen EW, Hla KM. Increased prevalence of sleep-disordered breathing in adults. Am J Epidemiol. 2013;177(9):1006-1014.

5. Fleetham J, Ayas N, Bradley D, et al. Canadian Thoracic Society guidelines: diagnosis and treatment of sleep disordered breathing in adults. Can Respir J. 2006;13(7):387-392.

6. Agustí A. Systemic effects of chronic obstructive pulmonary disease: what we know and what we don't know (but should). Proc Am Thorac Soc. 2007;4(7):522-525.

7. Agustí AG, Sauleda J, Miralles C, et al. Skeletal muscle apoptosis and weight loss in chronic obstructive pulmonary disease. Am J Respir Crit Care. 2002;166(4):485-489.

8. Barnes P, Celli B. Systemic manifestations and comorbidities of COPD Eur Respir J. 2009;33(5):1165-1185.

9. Mathers CD, Loncar D. Projections of global mortality and burden of disease from 2002 to 2030. PLoS Med. 2006;3(11):e442.

10. Flenley DC. Sleep in chronic obstructive lung disease. Clin Chest Med. 1985;6(4):651-661.

11. Sanders MH, Newman AB, Haggerty CL, et al; Sleep Heart Health Study. Sleep and sleep-disordered breathing in adults with predominantly mild obstructive airway disease. Am J Respir Crit Care Med. 2003;167(1):7-14.

12. Mermigkis C, Kopanakis A, Foldvary-Schaefer N, et al. Health-related quality of life in patients with obstructive sleep apnoea and chronic obstructive pulmonary disease (overlap syndrome). Int J Clin Pract. 2007;61(2):207-211.

13. He BT, Lu G, Xiao SC, et al. Coexistence of OSA may compensate for sleep related reduction in neural respiratory drive in patients with COPD. Thorax. 2017;72(3):256-262.

14. Poh TY, Mac Aogain M, Chan AK, et al. Understanding COPD-overlap syndromes. Expert Rev Respir Med. 2017;11(4):285-298.

15. Marin JM, Soriano JB, Carrizo SJ, Boldova A, Celli BR. Outcomes in patients with chronic obstructive pulmonary disease and obstructive sleep apnea: the overlap syndrome. Am J Respir Crit Care Med. 2010;182(3):325-331.

16. Lavie P, Herer P, Lavie L. Mortality risk factors in sleep apnoea: a matched case-control study. J Sleep Res. 2007;16(1):128-134.
17. CDC. NCHS Data Linked to NDI Mortality Files [cited July 17, 2017]. Available from: NCHS Data Linked to NDI Mortality Files. Available from: https://www.cdc.gov/nchs/data-linkage/mortality-public.htm. Accessed June 27, 2017.

18. Tsai J, Ford ES, Li C, Zhao G. Past and current alcohol consumption patterns and elevations in serum hepatic enzymes among US adults. Addict Behav. 2012;37(1):78-84.

19. Ford ES, Wheaton AG, Chapman DP, Li C, Perry GS, Croft JB. Associations between self-reported sleep duration and sleeping disorder with concentrations of fasting and 2-h glucose, insulin, and glycosylated hemoglobin among adults without diagnosed diabetes. Diabetes. 2014;6(4):338-350.

20. World Health Organization [webpage on the Internet]. Global Physical Activity Surveillance. Available from: http://www.who.int/ncds/ surveillance/steps/GPAQ/en/. Accessed June 27, 2017.

21. Ainsworth BE, Haskell WL, Herrmann SD, et al. 2011 compendium of physical activities: a second update of codes and MET values. Med Sci Sport Exerc. 2011;43(8):1575-1581.

22. CDC [webpage on the Internet]. National Health and Nutrition Examination Survey 2007-2008 Data Documentation, Codebook, and Frequencies [cited July 17, 2017]. Available from: https://wwwn.cdc. gov/Nchs/Nhanes/2007-2008/MCQ_E.htm. Accessed June 27, 2017.

23. Levey AS, Stevens LA, Schmid CH, et al; CKD-EPI (Chronic Kidney Disease Epidemiology Collaboration). A new equation to estimate glomerular filtration rate. Ann Intern Med. 2009;150(9):604-612.

24. Khan QJ, Fabian CJ. How I treat vitamin D deficiency. J Oncol Prac. 2010;6(2):97-101

25. Berry CE, Wise RA. Mortality in COPD: causes, risk factors, and prevention. COPD. 2010;7(5):375-382.

26. Kostikas K, Clemens A, Patalano F. Prediction and prevention of exacerbations and mortality in patients with COPD. Expert Rev Respir Med. 2016;10(7):739-753.

27. Sin DD, Anthonisen NR, Soriano JB, Agusti AG. Mortality in COPD: role of comorbidities. Eur Respir J. 2006;28(6):1245-1257.

28. Mansukhani MP, Wang S, Somers VK. Sleep, death, and the heart. Am J Physiol Heart Circ Physiol. 2015;309(5):H739-H749.

29. Bauters F, Rietzschel ER, Hertegonne KB, Chirinos JA. The link between obstructive sleep apnea and cardiovascular disease. Curr Atheroscler Rep. 2016;18(1):1.

30. King S, Cuellar N. Obstructive sleep apnea as an independent stroke risk factor: a review of the evidence, stroke prevention guidelines, and implications for neuroscience nursing practice. J Neurosci Nurs. 2016; 48(3):133-142.

31. Lyons OD, Bradley TD. Heart failure and sleep apnea. Can J Cardiol. 2015;31(7):898-908.

32. Adams RJ, Appleton SL, Vakulin A, et al. Chronic kidney disease and sleep apnea association of kidney disease with obstructive sleep apnea in a population study of men. Sleep. 2017;40(1).

33. Terzano C, Conti V, Di Stefano F, et al. Comorbidity, hospitalization, and mortality in COPD: results from a longitudinal study. Lung. 2010; 188(4):321-329.

34. Negewo NA, McDonald VM, Gibson PG. Comorbidity in chronic obstructive pulmonary disease. Respir Investig. 2015;53(6):249-258.

35. Stanchina ML, Welicky LM, Donat W, Lee D, Corrao W, Malhotra A. Impact of CPAP use and age on mortality in patients with combined COPD and obstructive sleep apnea: the overlap syndrome. J Clin Sleep Med. 2013;9(8):767-772.

36. Shawon MS, Perret JL, Senaratna CV, Lodge C, Hamilton GS, Dharmage SC. Current evidence on prevalence and clinical outcomes of co-morbid obstructive sleep apnea and chronic obstructive pulmonary disease: a systematic review. Sleep Med Rev. 2017;32:58-68.

37. McNicholas WT. Chronic obstructive pulmonary disease and obstructive sleep apnea: overlaps in pathophysiology, systemic inflammation, and cardiovascular disease. Am J Respir Crit Care Med. 2009; 180(8):692-700. 


\section{Publish your work in this journal}

The International Journal of COPD is an international, peer-reviewed journal of therapeutics and pharmacology focusing on concise rapid reporting of clinical studies and reviews in COPD. Special focus is given to the pathophysiological processes underlying the disease, intervention programs, patient focused education, and self management protocols.

This journal is indexed on PubMed Central, MedLine and CAS. The manuscript management system is completely online and includes a very quick and fair peer-review system, which is all easy to use. Visit http://www.dovepress.com/testimonials.php to read real quotes from published authors.

Submit your manuscript here: http://www.dovepress.com/international-journal-of-chronic-obstructive-pulmonary-disease-journal 